\title{
Multi-scale investigations of interface properties, stacking order and decoupling of few layer graphene on C-face 4H-SiC
}

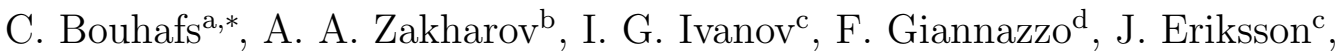 \\ V. Stanishev ${ }^{a}$, P. Kühne ${ }^{a}$, T. Iakimov ${ }^{c}$, T. Hofmann ${ }^{\mathrm{e}}$, M. Schubert ${ }^{\mathrm{e}}$, F.

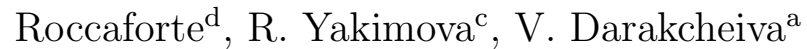 \\ ${ }^{a}$ Terahertz Materials Analysis Center, Department of Physics, Chemistry and Biology, \\ IFM, Linköping University,Linköping S-58183 SE, Sweden \\ ${ }^{b}$ MaxLab, Lund University, S-22100 Lund, Sweden \\ ${ }^{c}$ Department of Physics, Chemistry and Biology, IFM, Linköping University,Linköping \\ S-58183 SE, Sweden \\ ${ }^{d}$ CNR-IMM, Strada VIII, 5, 95121 Catania, Italy \\ ${ }^{e}$ Department of Electrical Engineering and Center for Nanohybrid Functional Materials, \\ University of Nebraska-Lincoln, Lincoln, Nebraska 68588, U.S.A
}

\section{Abstract}

In this work, we report a muti-scale investigation using several nano-, micro and macro- scale techniques of few layer graphene (FLG) sample consisting of large monolayer (ML) and bilayer (BL) areas grown on $\mathrm{C}$-face $4 \mathrm{H}-\mathrm{SiC}$ (000-1) by high-temperature sublimation. Single $1 \times 1$ diffraction patterns are observed by micro-low-energy electron diffraction for ML, BL and trilayer graphene with no indication of out-of-plane rotational disorder. $\mathrm{A} \mathrm{SiO}_{x}$ layer is identified between graphene and $\mathrm{SiC}$ by $\mathrm{X}$-ray photoelectron emission spectroscopy and reflectance measurements. The chemical composition of the interface layer changes towards $\mathrm{SiO}_{2}$ and its thickness increases with aging in normal ambient conditions. The formation mechanism of the inter-

${ }^{*}$ Corresponding author: Tel: 004613 285707. E-mail: vanya.darakchieva@liu.se

Preprint submitted to Journal of ${ }^{A} T_{E} X$ Templates

December 26, 2016

C) 2017. This manuscript version is made available under the Elsevier user license http://www.elsevier.com/open-access/userlicense/1.0/ 
face layer is discussed. It is shown by torsion resonance conductive atomic force microscopy that the interface layer causes the formation of non-ideal Schottky contact between ML graphene and $\mathrm{SiC}$. This is attributed to the presence of a large density of interface states. Mid-infrared optical Hall effect measurements revealed Landau-level transitions in FLG that have a squareroot dependence on magnetic field, which evidences a stack of decoupled graphene sheets. Contrary to previous works on decoupled C-face graphene, our BL and FLG are composed of ordered decoupled graphene layers without out-of-plane rotation.

\section{Introduction}

Graphene grown by sublimation on silicon carbide ( $\mathrm{SiC}$ ) offers a viable route towards production of large-scale electronic grade single-crystalline material on semi-insulating (SI) substrate without the need of transfer [1, 2, 3, 4,

5 [5, 6]. In particular, graphene grown on the C-face $(000 \overline{1})$ of $4 \mathrm{H}$ - and $6 \mathrm{H}-\mathrm{SiC}$ (C-face graphene) presents interest due to its high mobility parameters as compared to Si-face graphene (grown on the (0001) surface of 4H-, 6H-SiC) [7, 8, 9, 10, 11, 12. C-face graphene with free charge carrier mobility in the range of $5000-18000 \mathrm{~cm}^{2} / \mathrm{Vs}$ at room temperature has been demonstrated [8, 11, 12], which raises the hope to achieve epitaxial material with electronic properties matching those of exfoliated graphene transferred on either $\mathrm{SiO}_{2} / \mathrm{Si}[13$ ] or $\mathrm{BN}$ [14]. On the other hand, the room-temperature mobility parameters of Si-face graphene are significantly lower, 900-2400 $\mathrm{cm}^{2} / \mathrm{Vs}$ for as-grown [7, 9] and up to $3100 \mathrm{~cm}^{2} / \mathrm{Vs}$ for hydrogen intercalated quasi-freestanding graphene [15]. 
In contrast to Si-face graphene, which is extensively studied and well understood, the interface and structural properties of C-face graphene are not established. In all Si-face graphene, a buffer layer with $(6 \sqrt{3} \times 6 \sqrt{3}) \mathrm{R} 30^{\circ}$ surface reconstruction surface is always found at the interface with $\mathrm{SiC}$ independently of growth temperature, environment, pressure, number of layers etc. [16, 17]. The buffer layer has a graphene-like arrangement with mixed $\mathrm{sp}^{3}-\mathrm{sp}^{2}$ hybridization and it is strongly bonded to the substrate [16, 17]. On the other hand, very different interface properties and surface reconstructions are reported for $\mathrm{C}$-face graphene although it is generally accepted that it is weakly bonded to the substrate [18, 19]. For C-face graphene grown at relatively low temperatures (up to $1400^{\circ} \mathrm{C}$ ) in ultra-high vacuum, coexistence of $2 \times 2$ and $3 \times 3 \mathrm{SiC}$ surface reconstructions are reported for low graphene coverage [16, 19]. When grown at slightly higher temperatures of $1475^{\circ} \mathrm{C}$ and in Ar atmosphere $1 \times 1$ surface reconstruction [20] or a disordered interface 21] are observed. Mathieu et al. reported chemically heterogeneous interface layer for such graphene samples with at least two different SiC surface chemical states [20]. As a result, a local variation of C-face graphene workfunction was observed and attributed to different charge transfer from each of the $\mathrm{SiC}$ interfaces [20].

For C-face graphene grown by high-temperature sublimation in Ar atmosphere $\left(1850^{\circ} \mathrm{C}-2000^{\circ} \mathrm{C}\right)$ no specific $\mathrm{SiC}$ surface reconstruction has been reported [22]. We have recently shown that an amorphous layer consisting of a mixture of $\mathrm{C}$ and $\mathrm{Si}$ is formed between graphene and $\mathrm{SiC}$ for hightemperature growth in Ar atmosphere on mechanically polished 4H-SiC [23]. ${ }_{40}$ The thickness of the interface layer was shown to increase with increasing 
growth temperature and number of graphene layers [23]. The presence of an amorphous interface layer containing $\mathrm{Si}, \mathrm{C}$ and $\mathrm{O}$ was also reported for a similar C-face graphene sample grown by high-temperature sublimation in $\operatorname{Ar}$ [24. In this case, the interface layer had a constant thickness independent of graphene number of layers. For electronic device applications typically high-purity $\mathrm{SiC}$ substrates with chemically-mechanically polished (epi-ready) surfaces should be employed. Whether an interface layer would also occur in the case of C-face graphene grown on epi-ready surfaces of $\mathrm{SiC}$ without polishing defects is not known. The properties of the interface layer will have critical implications for the performance of $\mathrm{C}$-face graphene devices. Despite the numerous reports, very little is known about the effect of the interface layer on the electronic, transport and structural properties of C-face graphene.

In addition, there is an ongoing controversy about the nature of C-face graphene grown in Ar at relatively high temperatures [22, 21, 25]. It has been argued that a rotational disorder between the layers in few-layer graphene (FLG) stack leads to their decoupling, which can provide an explanation for the single $\pi$-band observed for C-face FLG in angle-resolved photoemission spectroscopy (ARPES) [18, 21]. On the other hand, Johansson et al. reported that rotation of different domains does not occur out-of-plane between adjacent layers in the stack when graphene is grown at high temperature, but rather in-plane within the same graphene sheet [22]. However, it was not clear if these ordered graphene sheets were decoupled or not. Recently, it was reported by nano-ARPES that C-face graphene grown at high temper65 ature in $\mathrm{Ar}$ atmosphere is $\mathrm{AB}$ stacked and the single $\pi$-band observed in 
ARPES experiments is a superposition of several multiple bands associated with two, three or more AB stacked layers [25]. The reasons behind decoupling of individual sheets in C-face graphene are not well understood and present an important scientific question from fundamental and technological points of view. The (de)coupling of C-face graphene also has crucial impact on its electronic properties and applications in electronic device. A stack of decoupled graphene sheets exhibits a Dirac-like band structure with linearly dispersing bands (Dirac cones) at the graphene K point, and free-charge carriers behave as massless Dirac fermions. In such a case, special transport properties arise from the chiral nature of free charge carriers leading to extremely high mobility parameters, ballistic transport, linear current-voltage characteristics, reduced back-scattering, electric field effect, anomalous quantum Hall effect, etc. On the other hand, a stack of coupled graphene layers exhibits a parabolic band structure with massive free charge carriers.

80 decoupling of C-face graphene with large homogeneous areas of monolayer (ML), bi-layer (BL) and tree-layer (TL) grown by high-temperature sublimation $\left(1950^{\circ} \mathrm{C}\right)$ on $4 \mathrm{H}-\mathrm{SiC}$. C-face graphene is a complex material and consists typically of multi-layer domains with varying thicknesses and/or properties, e.g. stacking order, doping, decoupling [26, 27]. Consequently, a multi-scale approach combining advanced experimental characterizations tools is needed to properly investigate and understand the properties of C-face graphene. We have combined several nanoscale (low-energy electron microscopy, Xray photo-electron emission microscopy, scanning Kelvin probe microscopy, 90 torsion conductive atomic force microscopy), microscale (low-energy electron 
diffraction, X-ray photo-electron spectroscopy, Raman scattering, reflectance mapping and linescan measurements) and macroscopic (mid-infrared optical Hall effect) characterization techniques to investigate the effect of the interface layer on the electrical properties and doping of graphene in correlation the standard Radio Corporation of America cleaning procedure, followed by dipping it into $\mathrm{HF}$ solution to remove the $\mathrm{C}$-face $\mathrm{SiC}$ surface native oxide.

The number of graphene layers and their distribution across the sample 
115 conductive atomic force microscopy (TR-CAFM) that was used to measure simultaneously topographic and current maps. TR-CAFM is a dynamic scanning probe method which allows non destructive electrical measurements 
from a conductive tip oscillating in a torsional or twisting mode in close proximity to the sample surface $(0.33 \mathrm{~nm})$. This non contact method has a distinct advantage over the conventional conductive atomic force microscopy (CAFM) performed in contact mode because of the absence of shear forces that can be damaging for the graphene sheets [30]. Measurements were carried out using a Veeco DI3100 AFM with Nanoscope V controller and tunneling atomic force microscopy (TUNA) application module. Platinumcoated Si tips with a typical apex radius of 10-20 nm were used. During a surface scan, a DC bias is applied between the tip and the sample backside and nanoscale resolution maps of the tunneling current flowing vertically through the graphene/SiC interface were collected. From a selected position of the current maps, a local $\mathrm{I}_{\mathrm{V}} \mathrm{V}_{\text {tip }}$ measurements were carried out by scanning current spectroscopy where the conductive tip is in direct contact with graphene. The local $\mathrm{I}-\mathrm{V}_{\text {tip }}$ measurements provide direct information on the electrical properties of the graphene/ $\mathrm{SiC}$ interface. It is worth noting that the contact between the metal tip and graphene is Ohmic, with a contact resistance that can be reduced down to few kohms by properly setting the tip/sample force. Furthermore, as will be evident from the experimental $\mathrm{I}-\mathrm{V}_{\text {tip }}$ characteristics, the semi-insulating $\mathrm{SiC}$ substrate introduces a series resistance contribution limiting the current flowing through graphene/SiC Schottky contact at higher bias values

The structural properties of graphene and their temporal evolution were studied by low energy electron microscopy (LEEM), low energy electron diffraction (micro-LEED), X-ray photoelectron emission microscopy (XPEEM) and X-ray photoelectron microspectroscopy (micro-XPS). The measurements 


\section{Results and discussion}

\subsection{Thickness distribution and ordering of FLG}

Based on a large number of thickness maps and line-scans obtained from reflectance, and LEEM images taken at different locations of the sample it 185 is determined that the C-face graphene consists predominantly of ML and BL graphene. Typical distributions of the number of graphene layers are exemplified in Figs. 1 1 and b, showing representative thickness line-scan and a map, respectively. The analysis of the number of graphene layers from 
the line-scan renders $45 \% \mathrm{ML}, 18 \%$ BL graphene, $3 \%$ bare substrate where graphene did not form, and the remaining part is FLG ( $\geq 3 \mathrm{ML})$. These results are further confirmed by the thickness mapping obtained from reflectance (Fig. 1b) and LEEM imaging (Figs. 2a-c). The ML and BL domains form predominantly as stripes (Figs. 1 b, $2 \mathrm{a}$ and $2 \mathrm{~b}$ ) following the $\mathrm{SiC}$ terraces and in large domains with constant thickness, e.g., with size as large as $\approx 500 \mu \mathrm{m}^{2}$ for the ML graphene in Fig. 2a. This is in contrast with previous reports on islanding of graphene with relatively small domain size when grown in Ar atmosphere [32, 33, 20]. A possible reason for these differences may be the fact that our graphene is grown at much higher temperature $\left(1950{ }^{\circ} \mathrm{C}\right)$ compared to conventional graphene grown in Ar atmosphere (below 1550 $\left.{ }^{\circ} \mathrm{C}\right)$ [32, 33, 20]. The higher growth temperature enables enhanced carbon diffusion and surface restructuring. We also note that the domain size with uniform thickness in our case is larger than previously reported for hightemperature sublimation C-face graphene [22, 23].
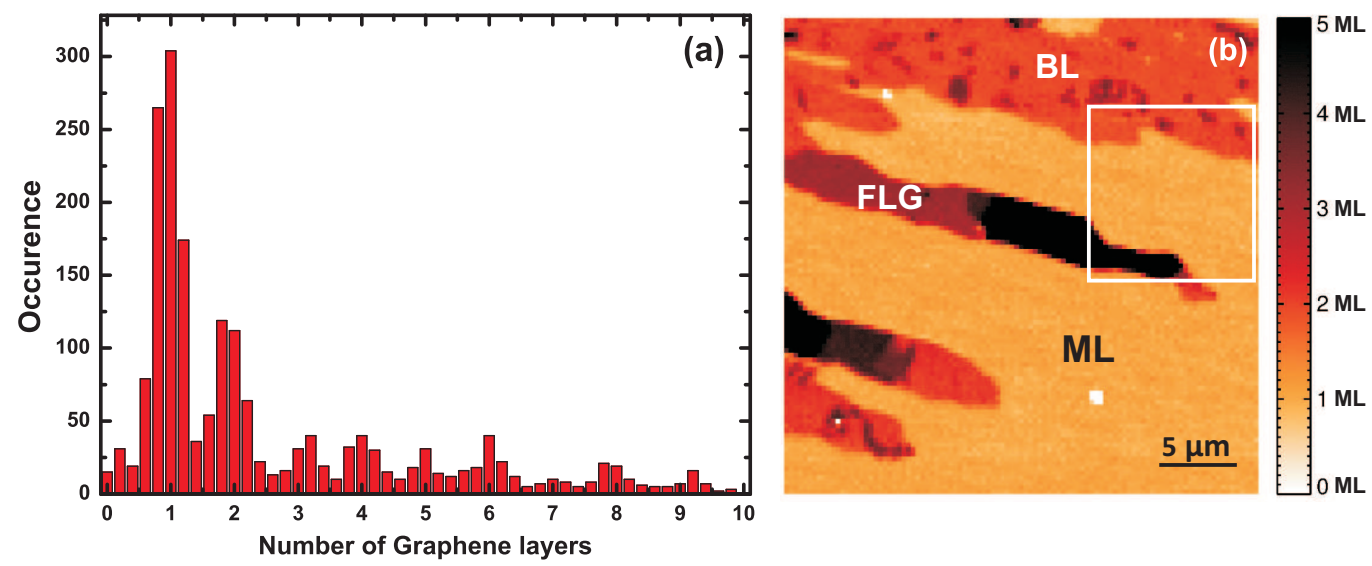

Figure 1: (a) Distribution of the number of graphene layers determined from a reflectance line scan of $2 \mathrm{~mm}$ length and (b) $30 \times 30 \mu \mathrm{m}^{2}$ thickness map obtained from the corresponding reflectance map. (A color version of this figure can be viewed online.) 
Representative LEEM images taken from different areas of the sample

The $\mu$-LEED patterns collected from the ML domain using an electron energy of $42 \mathrm{eV}$ shows single $1 \times 1$ diffraction pattern of graphene (indicated by $\vec{g}_{1}$ and $\vec{g}_{2}$ in Fig. $3($ a)) with unique azimuth orientation. The diffuse clouds 

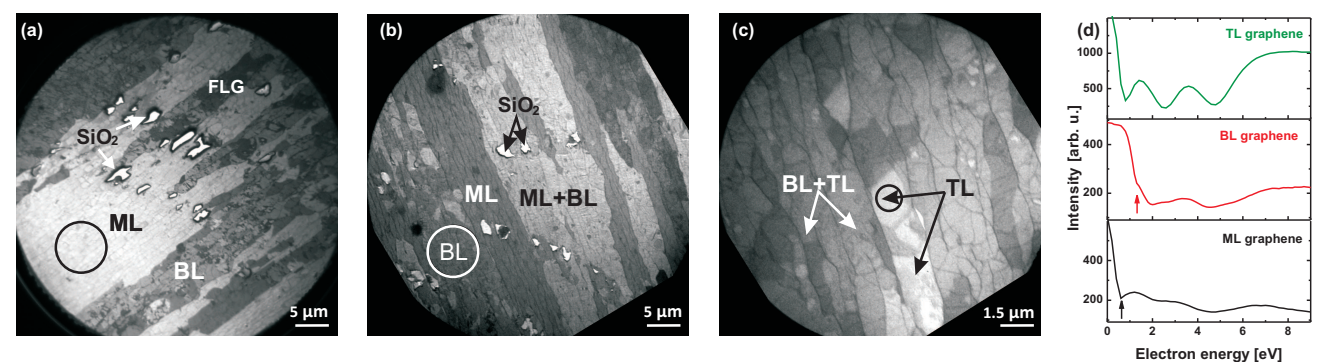

Figure 2: LEEM images taken at different locations of the sample and recorded with electron energy and a field of view of: (a) $1.5 \mathrm{eV}$ and $50 \mu \mathrm{m}$, respectively; (b) $1.5 \mathrm{eV}$ and $50 \mu \mathrm{m}$, respectively and (c) $5.6 \mathrm{eV}$ and $15 \mu \mathrm{m}$, respectively. Exemplary homogeneous ML, BL and TL graphene domains, as well as areas with mixed number of graphene, ML+BL and BL+TL, are indicated. (d) LEEM-I(V) curves taken from the homogeneous ML, BL and TL domains indicated with circles in (a), (b) and (c), respectively. (A color version of this figure can be viewed online.)

seen around the graphene diffraction spots can be caused by corrugation of the graphene layer or by a presence of an inhomogeneous interface layer. At higher electron energy of $74 \mathrm{eV}$ (Fig. $3(\mathrm{~d}))$ the six-fold $\mathrm{SiC} 1 \times 1$ diffraction spots (indicated by $\vec{S}_{1}$ and $\vec{S}_{2}$ ) become visible. The relative orientation of the graphene and $\mathrm{SiC}$ diffraction patterns shows that the single domain $\mathrm{ML}$ graphene is rotated by $\sim 12^{\circ}$ with respect to the substrate. Previously, Cface ML graphene was reported to be rotated by $30^{\circ}$ with respect to $\mathrm{SiC}$ [5]. Multiple or single domains of C-face graphene rotated by $\pm 1^{\circ}, \pm 7^{\circ}, \pm 10^{\circ}$ and $30^{\circ}$ with respect to $\mathrm{SiC} 1 \times 1$, have also been reported for adjacent layers within FLG. We note that a single graphene domain with unique azimuth orientation is observed in all ML regions in our sample. However, in some of these regions we could not resolve the $\mathrm{SiC}$ diffraction spots at higher electron energies, which might be related to a thicker interface layer. A similar effect is also observed in the BL regions of the sample - no diffraction patterns from $\mathrm{SiC}$ could be seen even at an electron energy of $80 \mathrm{eV}$ (Fig. 3(e)). 

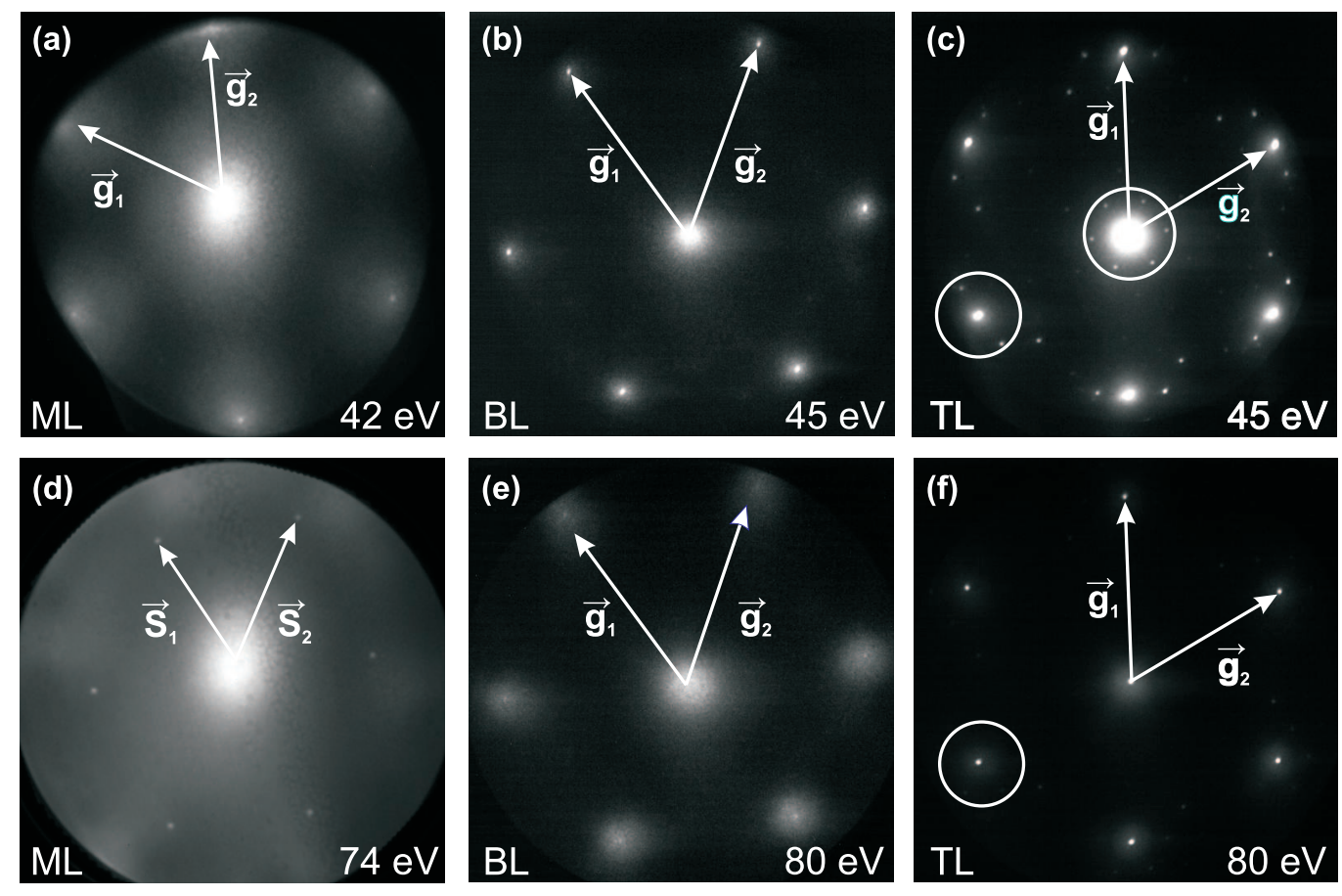

Figure 3: $\mu$-LEED diffraction patterns collected from the homogeneous regions indicated in Fig2(a) - (c) with circles: (a), (d) ML graphene area with a diameter of $4.5 \mu \mathrm{m}$ (indicated by a circle in Fig. 2a) and electron energies of (a) $42 \mathrm{eV}$ and (d) $78 \mathrm{eV}$; (b), (e) BL graphene area with a diameter of $4.5 \mu \mathrm{m}$ (indicated by a circle in Fig. 2p) and electron energies of (b) $45 \mathrm{eV}$ and (e) $80 \mathrm{eV}$; and (c), (f) TL graphene area with a diameter of $1 \mu \mathrm{m}$ (indicated by a circle in Fig. 22) and electron energies of (c) $45 \mathrm{eV}$ and (f) $80 \mathrm{eV}$. The reciprocal lattice vectors of graphene $\left(\vec{g}_{1}\right.$ and $\left.\vec{g}_{2}\right)$ and $\operatorname{SiC}\left(\vec{S}_{1}\right.$ and $\left.\vec{S}_{2}\right)$ are indicated in the figures. The circles in (c) and (f) highlight the superstructure diffraction spots. (A color version of this figure can be viewed online.)

The $\mu$-LEED diffraction pattern of BL graphene taken at $42 \mathrm{eV}$ (Fig. 3(b)) shows six graphene diffraction spots $\left(\vec{g}_{1}\right.$ and $\left.\vec{g}_{2}\right)$ similar to the case of ML graphene. Note that the single diffraction patterns observed in Figs. 3(b) and (e) indicate that there is no rotational disorder between the adjacent graphene layers in the BL graphene stack.

The $\mu$-LEED diffraction pattern of TL graphene collected using an electron energy of $45 \mathrm{eV}$ shows graphene $1 \times 1$ diffraction pattern similar to 
ML and BL graphene, and a superstructure (indicated by circles) around the spots of the 0 -order and $1 \times 1$ graphene diffraction pattern (Fig. 3(c)). Similar superstructure spots were previously observed for C-face TL graphene and suggested to be due to an interface layer akin to the buffer layer present in the

255 260 structure spots were never observed for BL graphene but could be found in the case of TL graphene grown on C-face 3C-SiC [34]. 


\subsection{Interface layer properties}

Figure 4(a) shows a C 1s XPEEM image taken at photon energy of 330 $\mathrm{eV}$ from the same region shown in Fig,2b. Representative $\mu$-XPS $\mathrm{C} 1 \mathrm{~s}$ and Si 2 p core-levels spectra taken from the homogeneous ML and BL graphene are shown in Figs. 4 (b) and (c), respectively. The C 1s and Si 2p spectra are measured with photon energy of $332 \mathrm{eV}$ and $200 \mathrm{eV}$, respectively. The $\mathrm{C}$
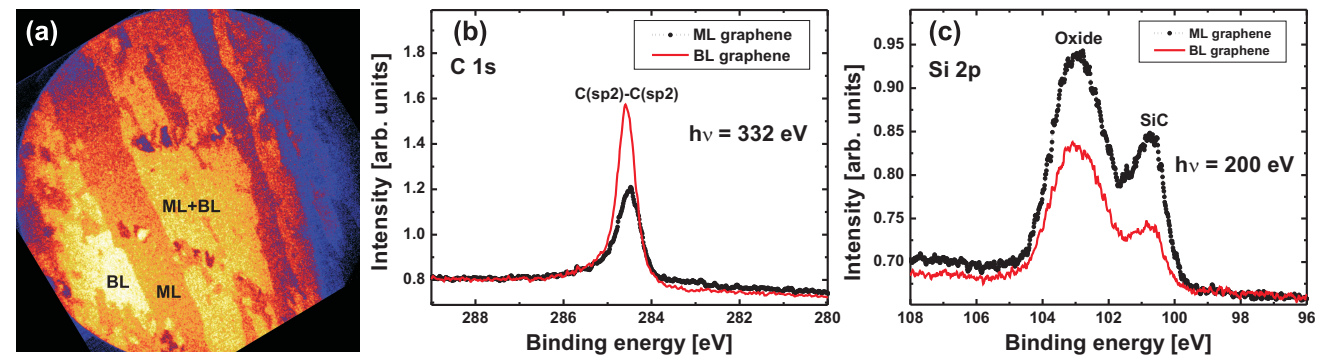

Figure 4: (a) C 1s XPEEM image with a field of view of $50 \mu \mathrm{m}$ taken at photon energy of $330 \mathrm{eV}$ with an electron energy of $41 \mathrm{eV} ; \mu$-XPS spectra of regions with different thickness: (b) C 1s peak measured using a photon energy $332 \mathrm{eV}$ for homogeneous ML and BL graphene; (c) Si 2p peak measured at $200 \mathrm{eV}$ for homogeneous ML and BL graphene. (A color version of this figure can be viewed online.)

1s core-level spectra of ML and BL graphene show the characteristic single narrow peaks associated with the $\mathrm{C}^{s p 2}-\mathrm{C}^{s p 2}$ bonds typical for $\mathrm{C}$-face graphene [16]. The $\mathrm{C}^{s p 2}-\mathrm{C}^{s p 2}$ peaks are located at $284.48 \mathrm{eV}$ and $284.6 \mathrm{eV}$ for ML and BL graphene, respectively. This result indicates that the work function of ML graphene is higher than for BL graphene, which can be related to different doping levels or/and different band structures of ML and BL graphene. Two well resolved peaks can be seen in the Si 2p core-level spectra of ML and BL graphene (Fig. 4(c)). The first peak located at $100.8 \mathrm{eV}$ originates from the $\mathrm{SiC}$ substrate. The second peak at $102.9 \mathrm{eV}$ can be associated with 
silicon to oxygen bonds, which we attribute to the presence of an interface $\mathrm{SiO}_{x}$ layer. The latter provides a possible explanation for the clouds observed in the graphene $\mu$-LEED diffraction pattern (Fig. 2(a)). The oxide peak is wide suggesting a broad distribution of oxidation states. No amorphous carbon could be identified at the interface between graphene and the substrate, as previously reported for C-face graphene grown by confinement controlled sublimation [20, 21] or on mechanically polished $4 \mathrm{H}-\mathrm{SiC}(000 \overline{1})$ by high temperature sublimation [23, 24].

In order, to investigate the effect of aging on the interface properties of graphene, Si 2P $\mu$-XPS spectra of ML graphene are measured after storing the sample for 24 months at room temperature in ambient conditions. The measurements were performed in a number of sample locations and similar results were obtained in all cases. Representative Si $2 \mathrm{P} \mu$-XPS spectra of as-grown ML graphene and ML graphene after 24 months of aging are shown in Fig. 5. It is clearly seen that the ratio between the $\mathrm{SiO}_{x}$ and the $\mathrm{SiC}$ peak intensities $\left[\mathrm{I}\left(\mathrm{SiO}_{x}\right) / \mathrm{I}(\mathrm{SiC})\right]$ increases with time. This is accompanied with a slight decrease of the peak separation suggesting that after 24 months the oxide composition is approaching $\mathrm{SiO}_{2}$ for ML graphene. The results indicate that the $\mathrm{SiO}_{x}$ interface is changing with time, its chemical composition gets closer to $\mathrm{SiO}_{2}$ and its thickness increases with aging at normal ambient conditions. The latter is further supported by reflectance measurements performed on the sample over a period of two years.

Fig. 6 shows the reflectance of the as-grown (a) and after 24 months of aging graphene (b) together with the bare $4 \mathrm{H}-\mathrm{SiC}$ substrate reference. The reflectance of as-grown ML graphene is $1.8 \%$ higher than the average re- 


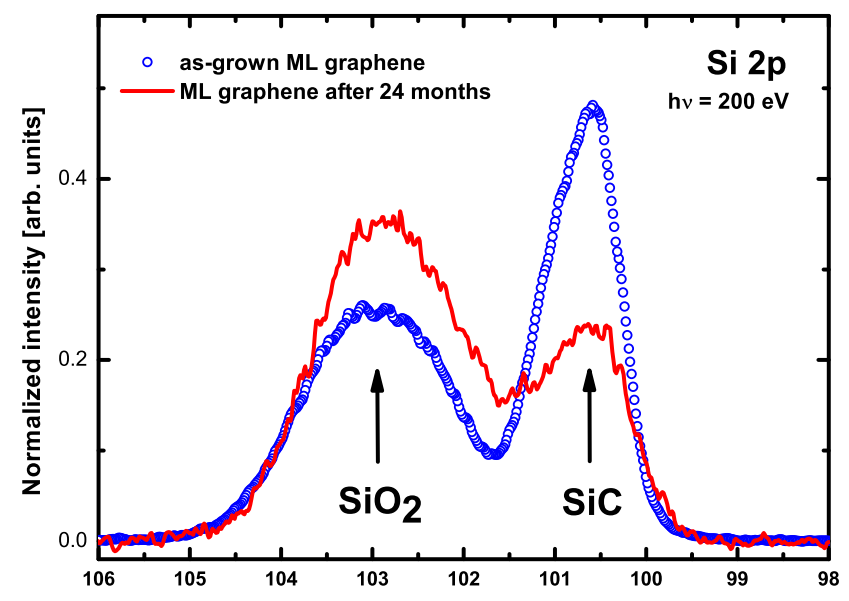

Figure 5: Representative Si 2p $\mu$-XPS spectra using a photon energy of $200 \mathrm{eV}$ of as-grown ML graphene (dots) and ML graphene after 24 months of sample storing under normal ambient conditions (line). (A color version of this figure can be viewed online.)

flectance of the bare $4 \mathrm{H}-\mathrm{SiC}(000 \overline{1})$ substrate in agreement with Ref. [29]. In contrast, the reflectance of ML graphene after 24 months of aging (Fig. 5(b)) is only $0.47 \%$ higher than the reference reflectance of bare $4 \mathrm{H}-\mathrm{SiC}$. Note that the difference in reflectance of two consecutive graphene layers, e.g., ML/BL and $\mathrm{BL} / \mathrm{TL}$, is always the same $-1.7 \%$. The observed differences in the reflectance of ML graphene with respect to the substrate for as-grown graphene 320 and after aging can be understood considering the presence of interface $\mathrm{SiO}_{x}$ layer. It is well known that native $\mathrm{SiO}_{2}$ may act as anti-reflective coating for a particular thickness. Therefore, the observed low reflectance of ML graphene after 24 months can be explained by an increased thickness of the $\mathrm{SiO}_{x}$ interface layer in agreement with the $\mu$-XPS results (Fig.5).

We suggest that the observed $\mathrm{SiO}_{x}$ at the interface between graphene and 

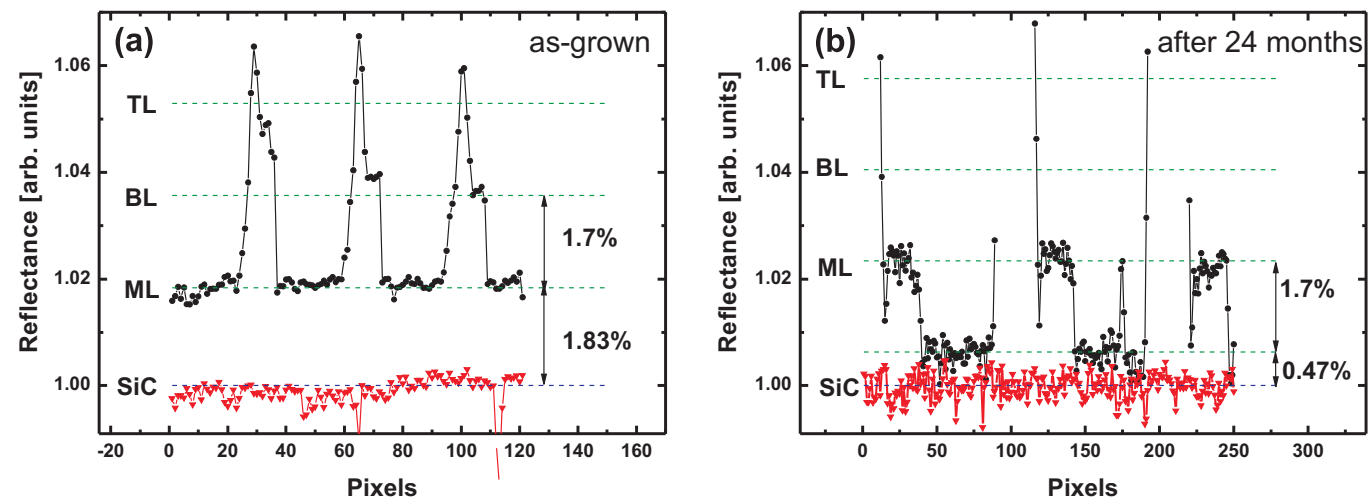

Figure 6: Reflectance of graphene (black dots) and a bare 4H-SiC substrate reference (red triangles): (a) as-grown and (b) after 24 months of aging. (A color version of this figure can be viewed online).

$\mathrm{SiC}$ is formed by oxidation of $\mathrm{Si}$ atoms trapped at the interface, which can be related to the specifics of the sublimation process. During the sublimation process, Si atoms are transported from the bulk of SiC to its surface followed by their desorption. As a result, a C-rich environment at the surface is created and which enables the graphene growth. The growth of subsequent graphene layers depends on the capability of the $\mathrm{Si}$ atoms to diffuse and desorb. After the formation of graphene, the desorption of Si atoms becomes difficult, which can lead to an excess of Si atoms trapped at the interface. Then Si oxidation might occur after graphene growth due to the presence of oxygen in the environment and as a result of exposure to normal ambient [35, 36]. This is consistent with the observed time evolution of the interface layer thickness. We believe that oxidation of $\mathrm{SiC}$ during growth is unlikely since $\mathrm{SiO}_{x}$ was reported to be stable up to a temperature of $1600{ }^{\circ} \mathrm{C}$ [26] while our growth temperature is $1950^{\circ} \mathrm{C}$. Our ML graphene shows a relatively high density of defects, which may facilitate the oxygen diffusion and oxidation of 
Si (See section 3.4: Doping and defects).

\subsection{Surface potential, conductivity mapping, and transport of FLG}

Kelvin probe measurements over areas of few mm gives an average graphene work function $W_{G}$ of about $5.00 \mathrm{eV}$, which is higher than the work function of undoped graphene $\left(W_{G N}=4.5 \mathrm{eV}\right)$ [37]. This indicates that the average doping of our graphene sample is $p$-type, which is also confirmed by terahertz OHE (not shown here). Local SKPM measurements show that ML graphene has a higher p-type doping compared to FLG (See section S.2 in the supplementary material). We use TR-CAFM to get insight in the mechanisms of current transport across the graphene/ $\mathrm{SiC}(000 \overline{1})$ interface. A representative AFM morphology and the corresponding current map both acquired in the torsion resonance mode are presented in Fig. 7(a) and (b), respectively. FLG areas (BL, TL and multilayers) can be identified by the presence of higher wrinkles than in the ML regions. Interestingly, from the current map in Fig. 7(b), a significantly lower current level can be observed in the ML graphene regions with respect to FLG. To investigate these electrical conduction differences through ML and FLG interfaces with $\mathrm{SiC}(000 \overline{1})$, local current-voltage characteristics ( $\mathrm{I}-\mathrm{V}_{t i p}$, with $\mathrm{V}_{\text {tip }}$ the tip bias) have been performed at several positions on the scanned area. Two representative $\mathrm{I}-\mathrm{V}_{\text {tip }}$ characteristics measured on the ML and FLG regions are shown in Fig. 7(c). A clear rectifying behavior is observed for the ML graphene contact to $\mathrm{SiC}(000 \overline{1})$, while symmetric $\mathrm{I}_{-} \mathrm{V}_{\text {tip }}$ characteristics are measured on the FLG regions. These striking differences in the vertical conduction can be ascribed, in a simple approximation, to the higher work function value of ML areas with respect to FLG detected by SKPM. 

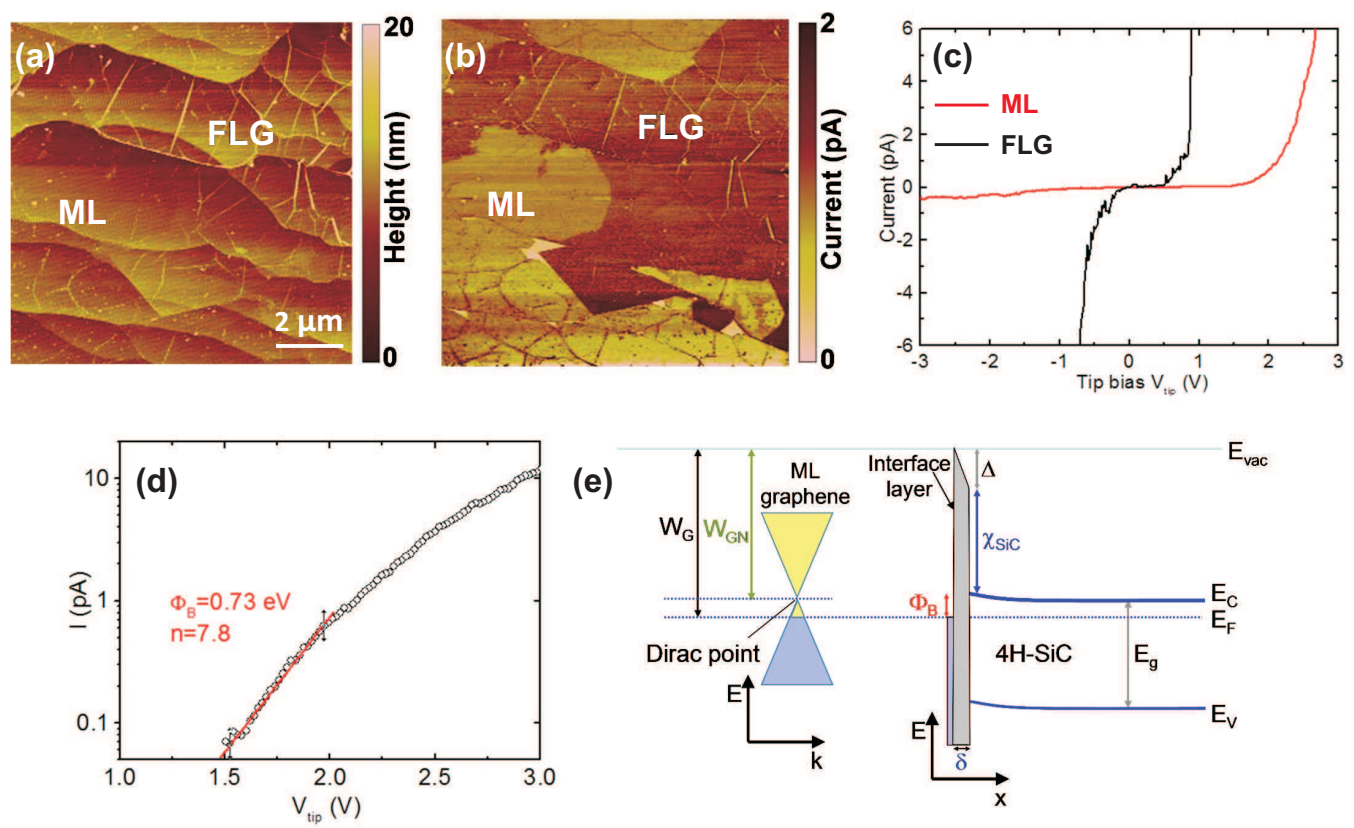

Figure 7: (a) TR-CAFM topography and (b) the corresponding current map of C-face graphene with ML and FLG regions indicated. (c) Representative I- $\mathrm{V}_{t i p}$ characteristics measured on the ML and FLG regions. (d) Semilog-scale plot of the local I-Vtip characteristic measured on ML graphene and fitting of the linear region with the thermionic emission law (the obtained Schottky barrier height $\phi_{B}=0.73 \mathrm{eV}$ and ideality factor $\mathrm{n}=7.8$ are indicated). (e) Schematic band diagram of the ML/ $\mathrm{SiC}(000 \overline{1})$ contact with a $\mathrm{SiO}_{x}$ interface layer with a thickness $\delta$ which accounts for the difference $\Delta=1.2 \mathrm{eV}$ between the experimental and ideal SBH values. (A color version of this figure can be viewed online.)

In general, for an ideal graphene/SiC Schottky contact (no interface layer exists between graphene and $\mathrm{SiC}$ ), the Schottky barrier height ( $\mathrm{SBH})$ is expected to increase with graphene work function resulting in a reduced thermionic emission current above the barrier. However, in our graphene sample a substoichiometric $\mathrm{SiO}_{x}$ layer between graphene and $\mathrm{SiC}$ is present as inferred by $\mu$-LEED, $\mu$-XPS and reflectance analyses. The presence of such an interface layer is expected to play a key role in the graphene/SiC contact properties causing a deviation from the ideal Schottky-Mott behav- 
ior. To extract more quantitative information on the contact properties, the local I- $\mathrm{V}_{t i p}$ characteristic measured on the ML graphene region is reported in Fig. $7($ d) on a semilog-scale and the fitting of the linear region with the thermionic emission law is carried out. The obtained values of the SBH and ideality factor are $\phi_{B}=0.73 \mathrm{eV}$ and $n=7.8$, respectively. The experimental $n$ value is much higher than unity and clearly indicates that the $\mathrm{ML} / \mathrm{SiC}(000 \overline{1})$ contact cannot be described as an ideal Schottky contact. Furthermore, the obtained $\mathrm{SBH}$ value for graphene on $\mathrm{SiC}(000 \overline{1})$ is much lower than the ideal one expected according to the Schottky-Mott relation $\phi_{B}=W_{G^{-}} \chi=1.9 \mathrm{eV}$, being $W_{G}=5 \mathrm{eV}$ the average graphene work function measured by Kelvin probe measurements and $\chi=3.1 \mathrm{eV}$ the $4 \mathrm{H}-\mathrm{SiC}$ electron affinity. These deviations from the ideal behavior can be described according to the Card-Rhoderic model for metal/semiconductor junctions with an interfacial layer [38], considering the effect of the $\mathrm{SiO}_{x}$ layer with thickness $\delta$, which introduces a density of interface states $\mathrm{D}_{i t}$ between graphene and $\mathrm{SiC}$. Fig. 7(e) shows a schematic band diagram of the ML graphene/SiC contact with this thin insulating layer, which accounts for the difference $\Delta=1.2 \mathrm{eV}$ between the experimental and ideal SBH values. Furthermore, the strong deviation of the ideality factor from unity can be explained in terms of a high interface-states density $\mathrm{D}_{i t}$, according to the relation [38]:

$$
n=1+\frac{e \delta D_{i t}}{\epsilon_{i} \epsilon_{0}}
$$

where $\epsilon_{i}$ and $\epsilon_{0}$ are $\mathrm{SiO}_{x}$ dielectric constant and the vacuum permittivity, respectively. Assuming a dielectric constant of 3.9 [39] for the $\mathrm{SiO}_{2}$ interface layer, we estimate a linear density of interface defects $\delta D_{i t}=1.47 \times 10^{7}$ 
$\mathrm{cm}^{-1} \mathrm{~V}^{-1}$

370

\subsection{Doping and defects of FLG}

More detailed information on doping of FLG can be obtained from the $\mu \mathrm{RS}$ analysis. Figs. $8(\mathrm{a})$ and (b) show representative $\mu \mathrm{RS}$ spectra taken from ML and BL graphene regions, where the D, G, D' and 2D peaks associated with graphene can be resolved [35. In addition, a peak in the vicinity of the $\mathrm{G}$ peak is also detected in the $\mu \mathrm{RS}$ spectra of ML graphene. This peak, hereafter referred to as the B peak, is located around $1590 \mathrm{~cm}^{-1}$ on the lowenergy side of the $\mathrm{G}$ peak (see Fig. 8b). In BL graphene no B peak can be resolved. Therefore, the Raman spectra in the vicinity of the G peak are fitted with three Lorentzian lineshapes accounting for the B, G and D' peaks in ML graphene, and using two Lorentzian lineshapes accounting for the $\mathrm{G}$ and $\mathrm{D}^{\prime}$ peaks in the case of BL graphene, respectively. The $\mathrm{D}$ and the 2D peaks are fitted each with a single Lorentzian lineshape for all graphene layers. (See Fig.S3 for the full width at half maximum (FWHM), of G and 2D peaks, and the B peak intensity in supplementary material)

It is seen from Fig. 8 that the G-, $\omega_{G}$, and the 2D-, $\omega_{2 D}$, peak positions for both ML and BL graphene are blue-shifted with respect to the corresponding positions for freestanding graphene [40] with $\omega_{G, 0}=1582 \mathrm{~cm}^{-1}$ and $\omega_{2 D, 0}=2670 \mathrm{~cm}^{-1}$. The observed shifts might be due to a compressive biaxial strain and/or doping. The biaxial strain results from a difference in thermal expansion coefficients of graphene and $\mathrm{SiC}$. The doping is due to a charge transfer from the $\mathrm{SiC}$ substrate and/or impurity incorporation [41, 42]. The 

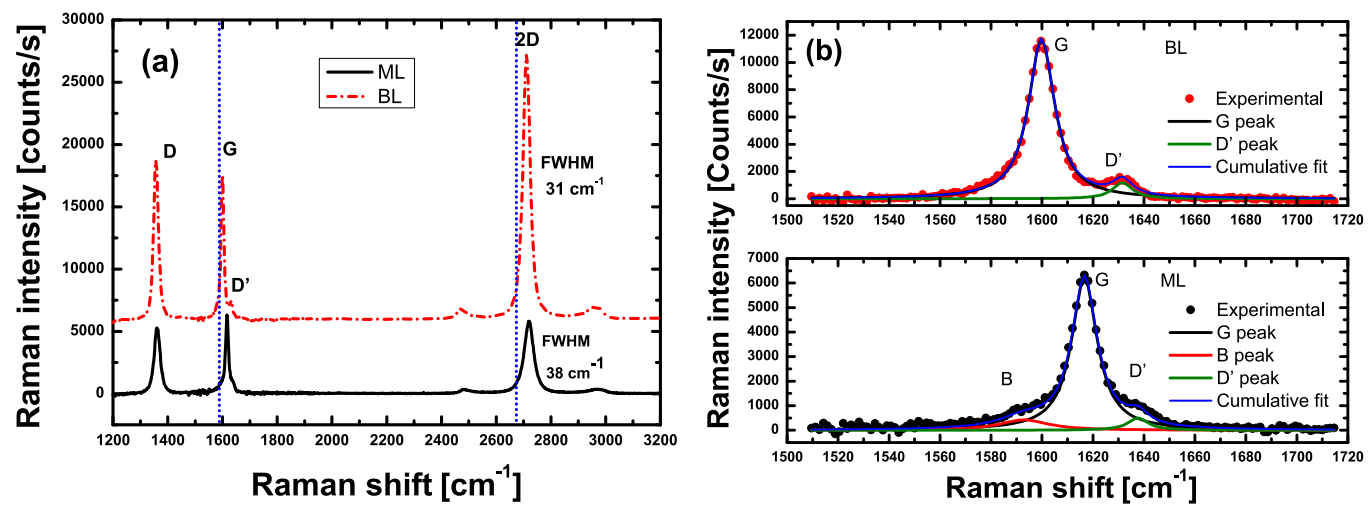

Figure 8: (a) Representative $\mu \mathrm{RS}$ spectra of ML (black solid line) and BL (red dashed line) graphene. The dotted lines indicate the $\mathrm{G}$ and $2 \mathrm{D}$ peaks positions of freestanding graphene $\left(\omega_{G, 0}=1582 \mathrm{~cm}^{-1}\right.$ and $\left.\omega_{2 D, 0}=2670 \mathrm{~cm}^{-1}\right)$. (b) Enlarged Raman spectra from (a) in the vicinity of the $\mathrm{G}$ peak. (A color version of this figure can be viewed online.)

G (2D) peak position shift due to biaxial strain $(\varepsilon)$ is described by:

$$
\Delta \omega_{G(2 D)}^{b}=-2 \omega_{G(2 D), 0} \gamma_{G(2 D)} \varepsilon
$$

where $\gamma_{G(2 D)}$ is the Grüneisen parameter of the $\mathrm{G}(2 \mathrm{D})$ peak. We use $\gamma_{G}=$ 1.8 and $\gamma_{2 D}=2.7$ [43, 44] resulting in a $\left(\Delta \omega_{2 D} / \Delta \omega_{G}\right)^{b}$ ratio of 2.53 as shown by a line in Fig. 9, referred hereafter as the biaxial strain line.

Figure. 9 shows a plot of the 2D versus $\mathrm{G}$ peak positions for ML (dots) and $\mathrm{BL}$ (triangles) graphene. The shifts of $\mathrm{G}$ and $2 \mathrm{D}$ peak positions for $\mathrm{ML}$ graphene with respect to the biaxial strain line indicates that ML graphene is highly doped. These results are in agreement with the SKPM observations (see section S.2 in supplementary material). On the other hand, the G and 2D peak positions for BL graphene are very close to the biaxial strain line implying very low doping levels in this case. The former is further confirmed by comparing the ratio between $2 \mathrm{D}$ and $\mathrm{G}$ peak intensities, $\mathrm{I}(2 \mathrm{D}) / \mathrm{I}(\mathrm{G})$. BL 
graphene shows a higher 2D peak intensities compared to the $\mathrm{G}$ peak, which is a signature of low doped graphene [45]. In contrast, ML graphene has much lower $\mathrm{I}(2 \mathrm{D}) / \mathrm{I}(\mathrm{G})$ ratios indicating a higher free charge carrier concentration (see Fig. 8) [45].

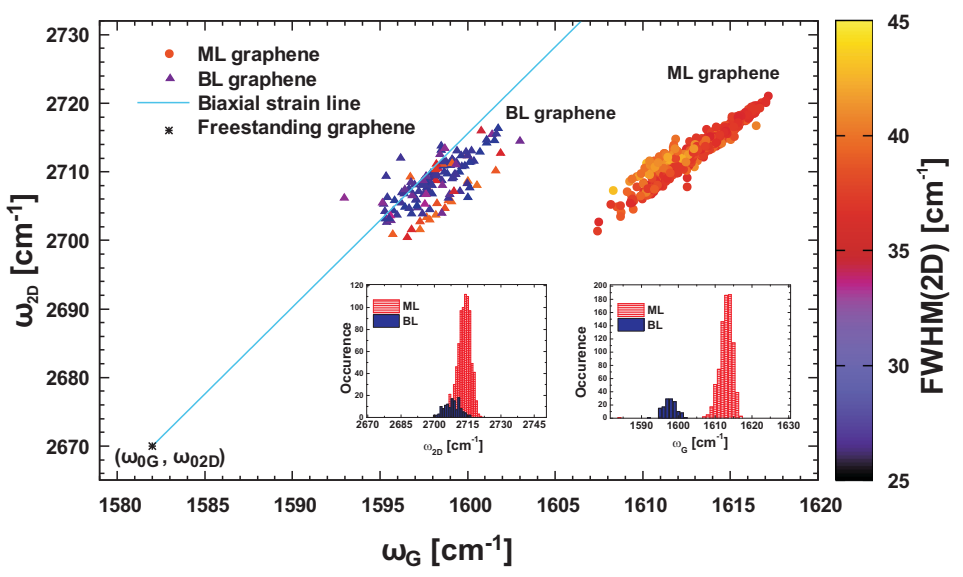

Figure 9: Experimental 2D peak position, $\left(\omega_{2 D}\right)$, versus G peak position, $\left(\omega_{G}\right)$, for ML (dots) and BL (triangles) graphene. The blue solid line represents the shifts due to biaxial compressive strain according the theoretical ratio $\left(\left(\Delta \omega_{2 D} / \Delta \omega_{G}\right)^{b}=2.53\right)$. The $G$ and $2 \mathrm{D}$ positions of freestanding graphene $\left(\omega_{G 0}\right.$ and $\left.\omega_{2 D 0}\right)$ are indicated by an asterisk. The histograms of the G peak and 2D peak positions for ML and BL graphene are shown in the insets. The color bar represents the 2D peak FWHM. (A color version of this figure can be viewed online.)

The free hole concentration in ML graphene is estimated from the experimental shifts of the $\mathrm{G}$ and $2 \mathrm{D}$ peaks with respect to free-standing graphene $\Delta \omega_{G(2 D)}$ assuming that they are linear combination of the respective shifts due to biaxial strain, $\Delta \omega_{G(2 D)}^{b}$, and doping, $\Delta \omega_{G(2 D)}^{h}$ [46]:

$$
\Delta \omega_{G(2 D)}=\Delta \omega_{G(2 D)}^{h}+\Delta \omega_{G(2 D)}^{b}
$$

Equation 3 is solved taking into account Eq2 and assuming $\gamma_{G}=1.8$ and $\gamma_{2 D}=2.7$ [43, 44], $\delta \omega_{G}^{h} / \delta \mathrm{E}_{F}=42 \mathrm{~cm}^{-1} \mathrm{eV}^{-1}$ and $\delta \omega_{2 D}^{h} / \delta \mathrm{E}_{F}=32 \mathrm{~cm}^{-1} \mathrm{eV}^{-1}$ 
reported for gated pristine graphene [45]. The average Fermi energy for ML graphene is $0.480 \mathrm{eV}$ and the respective average free hole concentration is $1.4 \times 10^{13} \mathrm{~cm}^{-2}$ (See Fig.S3(e) in the supplementary material for the free hole density map of ML graphene).

The free hole concentration can be anti-correlated with the B peak intensity - the lower the free hole concentration the higher the B peak intensity (see Fig. S3(d) and (e) in the supplementary data). The B peak observed in the Raman spectra of ML graphene at $1590 \mathrm{~cm}^{-1}$ has not been reported before. We speculate that the B peak might be due to the presence of small BL domains in the ML graphene regions. This is based on the XPEEM results showing ML graphene regions containing inclusions of BL graphene and considering the lower doping of BL compared to ML graphene. Furthermore, the $B$ peak position seems to be very close to the $G$ peak position of $B L$ 415 graphene (see Fig. S3(b) in the supplementary data). Such BL inclusions could be expected to generate defects in the ML regions where they occur. Indeed, a correlation between the B peak intensity and defect density is observed (See Fig. S3(f) in the supplementary material). A defect density of the order of $10^{11} \mathrm{~cm}^{-2}$ is estimated for ML and BL graphene (See Fig.S3(f) in the supplementary material for the defect density map of ML graphene), which is in line with our speculation that defects may be a potential path for oxygen diffusion after growth causing the formation of the observed $\mathrm{SiO}_{x}$ interface layer. Dislocations, disclinations and boundary defects generated at the grain boundaries [47], as a result of the three-dimensional growth of C-face graphene, are potential candidates to explain the origin of the large defect density in ML and BL graphene observed here. 


\subsection{Decoupling of individual layers in FLG}

Analysis of the Raman 2D peak shape also allows to draw conclusion about the coupling of the adjacent layers in FLG. In general, the 2D peak shape and FWHM depend on the number of graphene layers and their coupling following the change of electronic band structure [48]. For example, exfoliated ML graphene on $\mathrm{SiO}_{2} / \mathrm{Si}$ shows a symmetric 2D peak with Lorentzian shape and a $\mathrm{FWHM} \simeq 24 \mathrm{~cm}^{-1}$, while coupled AB-stacked BL graphene shows a broader and asymmetric $2 \mathrm{D}$ peak with a $\mathrm{FWHM} \simeq 55 \mathrm{~cm}^{-1}$, and can be fitted with four Lorentzian lineshapes [48]. C-face graphene typically consists of decoupled graphene layers, i.e., the graphene stack exhibits the same electronic band structure as that of a ML graphene. Hence, the 2D peak of decoupled FLG, similarly of the 2D peak of ML graphene, can be fitted with a single Lorentzian lineshape with FWHM in the range 24 $40 \mathrm{~cm}^{-1}$ [49]. The 2D peak of our ML and BL graphene has a symmetric Lorentzian lineshape with average FWHM of $39 \mathrm{~cm}^{-1}$ and $31 \mathrm{~cm}^{-1}$ for ML and BL, respectively (see Fig. S3(c) in the supplementary data). This result indicates that the graphene layers in the BL stack are decoupled. The somewhat broader 2D peak of ML graphene compared to BL graphene may be explained by the higher hole doping of the former as inferred by $\mu \mathrm{RS}$ and SKPM. An increase of the 2D FWHM with increasing doping concentration was previously reported and attributed to free charge carrier interactions [50, 51]. We note that TL graphene also shows a 2D peak with an average FWHM below $40 \mathrm{~cm}^{-1}$ indicating that the graphene layers in the TL graphene stack are also decoupled.

Further insight into the interaction between the adjacent graphene lay- 

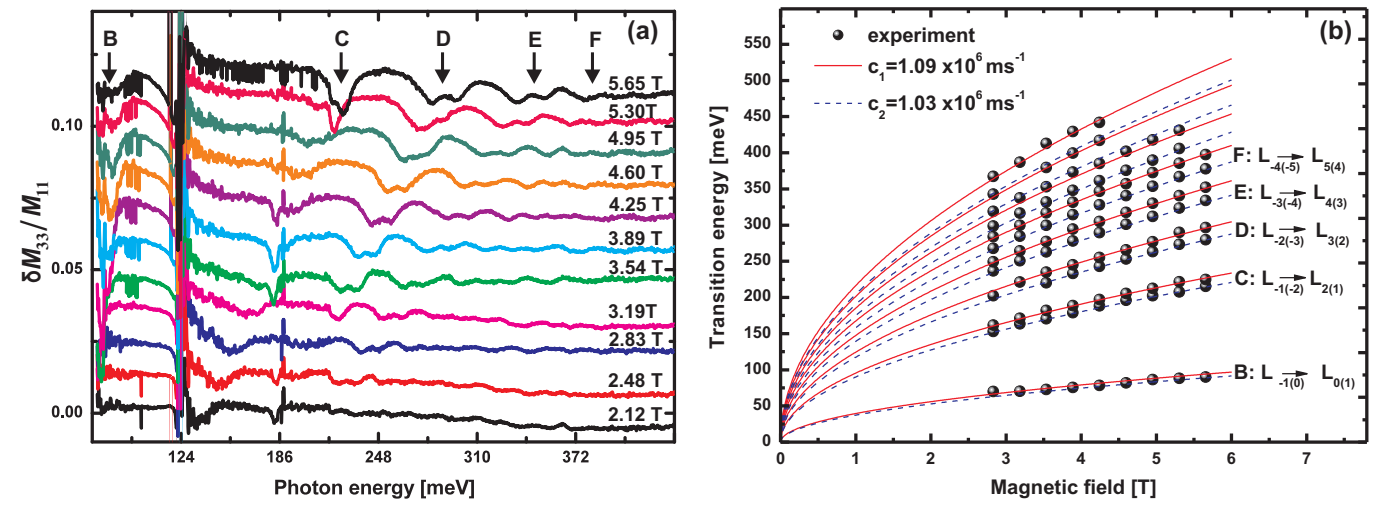

Figure 10: (a) On-diagonal block $\delta M_{33} / M_{11}$ optical Hall effect spectra for different values of the magnetic field. The spectra are measured at $1.5 \mathrm{~K}$ and shifted by 0.01 for clarity. (b) LL transition energy positions as a function of magnetic field. The blue dashed and red solid lines represent calculated transition energies for single layer graphene with Fermi velocities of $\tilde{\mathrm{c}}_{1}=1.03 \times 10^{6} \mathrm{~m} . \mathrm{s}^{-1}$ and $\tilde{\mathrm{c}}_{2}=1.09 \times 10^{6} \mathrm{~m} . \mathrm{s}^{-1}$, respectively. (A color version of this figure can be viewed online.)

ers in the graphene stack can be gained from inter-Landau-level (LL) spectroscopy. It is well established that ML graphene and stacks of decoupled graphene layers show a characteristic square-root dependence of the LL transition energies on magnetic field [52]. On the other hand, coupled AB BL, TL etc. graphene show sublinear dependence of the LL transitions on magnetic field [53]. We have recently shown that LL transitions can be measured by mid-infrared optical Hall effect (OHE) in oblique-incidence reflection mode and that single layer graphene or a stack of decoupled graphene layers exhibit transitions with polarization preserving selection rules while those for coupled graphene layers have polarization-mixing character [54]. In our graphene sample we observe LL transitions only in the block-on diagonal Mueller matrix element spectra indicating polarization preserving selection rules of the transitions and thus decoupled graphene layers in the

FLG stack [54]. Fig. 10](a) shows exemplary OHE difference spectra for one 
of the block on-diagonal Mueller matrix elements, $\delta M_{33} / M_{11}$, and for different magnitudes of the magnetic field. The LL transitions are indicated with letters following the notations of Sadowski et al. [52]. Note that LL transitions from ML graphene in our case cannot be detected due to the graphene $\left(E_{F}=0.480 \mathrm{eV}\right)$ even for the highest magnetic field of $5.66 \mathrm{~T}$ used in our MIR OHE experiments, the LL transitions from ML graphene occur outside the measured spectral range. Therefore, all measured LL transitions in our sample must originate from BL, TL and FL graphene, which show a 475 
field, as well as the symmetric shape of the Raman 2D peak with an average FWHM below $40 \mathrm{~cm}^{-1}$ indicate that adjacent layers in our BL, TL and FLG stacks are decoupled. Such behavior was previously observed for C-face graphene and attributed to rotational disorder of the adjacent graphene layers in the stack [18, 21]. However, the $\mu$-LEED patterns taken from different BL and TL regions of our sample show single $1 \times 1$ diffraction pattern of graphene (Fig. 3) with no out-o-plane rotation. Thus, our results indicate that BL, TL, and FL graphene are composed of decoupled graphene layers but are not rotationally disordered. The origin of layer decoupling in this case needs to be further investigated. A possible explanation is trapping of impurity atoms, such as $\mathrm{Si}$ or O between the layers in the stack, which may cause an increase of layer spacing and their electronic decoupling.

Our results on the decoupling of C-face FLG grown by high-temperature sublimation are in contrast with a recent report on AB stacked C-face graphene grown at $1900^{\circ} \mathrm{C}[25]$. Note that the decoupling in our case is inferred based on MIR OHE measurements that are probing the whole area of the sample and based on a large sampling of $\mu \mathrm{RS}$ maps across the sample. In contrast, the AB stacking order in [25] is inferred based on nano-ARPES measurements probing limiting area of the FLG. It is well known that C-face graphene consists of domains with multi-layers with varying thicknesses as also evidenced in this work [26, 27, 22, 20]. Furthermore, substrate defects may cause the formation of graphitic $\mathrm{AB}$ stacked inclusions within decoupled graphene stacks [54]. For example, we have previously shown that when grown on $\mathrm{SiC}$ substrate with polishing defects $\mathrm{C}$-face graphene is predominantly $\mathrm{AB}$ stacked [23]. $\mathrm{AB}$ stacking was also previously evidenced in the 
Raman spectra of C-face graphene nucleated at dislocations [56]. Therefore, nanoscale characterization techniques, which provide local information, may be best augmented with macroscopic techniques to draw comprehensive conclusions about the nature of C-face graphene, which is typically highly inhomogeneous

\section{Summary}

The interface structure and its implications for electrical transport, stacking order and decoupling of FLG on C-face 4H-SiC (0001) are investigated using multi-scale advanced characterization tools. Graphene is grown by high-temperature sublimation in argon atmosphere at optimal conditions to achieve large-area domains (few hundreds of $\mu \mathrm{m}^{2}$ ) with uniform ML, BL and TL graphene. However, fewer regions where graphene grows as mixtures of $\mathrm{ML}+\mathrm{BL}$ and $\mathrm{BL}+\mathrm{TL}$ with significantly smaller domain sizes, as well as thicker regions with $\geq 3$ MLs could also be found. Single $1 \times 1$ diffraction pattern is revealed by $\mu$-LEED for ML, BL and TL graphene. We find that the large-area uniform ML graphene is a single crystallographic grain with unique azimuth rotation rotated by $\approx 12^{\circ}$ with respect to SiC. Furthermore, BL and TL graphene do not show out-of-plane rotational disorder of the adjacent layers in the stack, which is in agreement with previous results for high-temperature C-face graphene [22].

An interface $\mathrm{SiO}_{x}$ layer is identified between C-face graphene and $\mathrm{SiC}$ independently of graphene thickness and domain size. We suggest that the $\mathrm{SiO}_{x}$ layer forms after growth as a result of oxidation of trapped $\mathrm{Si}$ atoms during the sublimation process, and speculate that defects in ML and BL 
540

graphene serve as diffusion paths for oxygen. We find that the chemical composition of the interface layer changes towards $\mathrm{SiO}_{2}$ and its thickness increases with aging in normal ambient conditions. TR-CAFM investigations show that the presence of the interface layer causes the formation of non-ideal Schottky contact between ML graphene and $\mathrm{SiC}$, which is associated with a large density of interface defects.

A correlation between SKPM and $\mu \mathrm{RS}$ shows that ML graphene is highly $p$-type doped with an average hole density of $1.4 \times 10^{13} \mathrm{~cm}^{-2}$ while BL and FL graphene have much lower free-charge carrier concentration below $10^{12} \mathrm{~cm}^{-2}$. These results are consistent with vertical current transport measurements across graphene/SiC interface by TR-CAFM mapping and local currentvoltage analyses.

Mid-infrared optical Hall effect measurements revealed Landau-level transitions in FLG that have a square-root dependence on magnetic field, which is characteristic for single layer graphene. This allows us to conclude that FLG consists of decoupled layers, which is further confirmed by $\mu$ RS analysis. Contrary to previous works on decoupled C-face graphene, our $\mu$-LEED investigations indicate that the adjacent layers in the BL and FLG stack are not rotationally disordered. Our results imply that the nature of C-face graphene is rather complicated and depends on growth conditions, substrate preparation, temperature, etc., in contrast to epitaxial graphene on Si-face $\mathrm{SiC}(0001)$ that shows coupled graphene layers with AB stacking, rather independent of growth conditions. Our findings on the interface layer and its implications for the electrical properties of FLG suggest that C-face ML and BL graphene grown by high-temperature sublimation are not optimal for de- 
565 adjacent layers have better perspective for future electronic applications.

\section{Acknowledgements}

The authors would like to acknowledge financial support from Marie Curie actions under Project No.264613-NetFISiC, the Swedish Research Council 570 (VR Contract 2013-5580), the Swedish Governmental Agency for Innovation Systems (VINNOVA) under the VINNMER international qualification program Grant No. 2011-03486, the Swedish foundation for strategic research (SSF) under Grants No. FFL12-0181 and No. RIF14-055, the Swedish Government Strategic Research Area in Materials Science on Functional Mate-

rials at Linköping University (Faculty Grant SFO Mat LiU No 2009 00971) and the FP7 EU project Nano-Rf under the Grant Agreement No. FP7-ICT2011-8. The research leading to these results has partly received funding from the European Union Seventh Framework Programme under Grant Agreement No. 604391 Graphene Flagship. This work was supported in part by

the National Science Foundation (NSF) through the Center for Nanohybrid Functional Materials (EPS-1004094), the Nebraska Materials Research Science and Engineering Center (DMR-1420645), and awards CMMI 1337856 and EAR 1521428.

\section{References}

${ }_{585}^{2}$ [1] Berger C, Song Z, Li T, Li X, Ogbazghi A, Feng R, et al. Ultrathin Epitaxial Graphite: 2D Electron Gas Properties and a Route toward 
Graphene-based Nanoelectronics. The Journal of Physical Chemistry B. 2004;108(52):19912-19916. doi:http://dx.doi.org/10.1021/jp040650f.

[2] Berger C, Song Z, Li X, Wu X, Brown N, Naud C, et al. Electronic Confinement and Coherence in Patterned Epitaxial Graphene. Science. 2006;312(5777):1191-1196. doi:http://dx.doi.org/10.1126/science.1125925.

[3] Virojanadara C, Syväjarvi M, Yakimova R, Johansson LI, Zakharov AA, Balasubramanian T. Homogeneous large-area graphene layer growth on 6H-SiC(0001). Phys Rev B. 2008;78:245403. doi:http://dx.doi.org/10.1103/PhysRevB.78.245403.

[4] Emtsev KV, Bostwick A, Horn K, Jobst J, Kellogg GL, Ley L, et al. Towards wafer-size graphene layers by atmospheric pressure graphitization of silicon carbide. Nature Materials. 2009;8(3):203-207. doi:http://dx.doi.org/10.1038/NMAT2382.

[5] de Heer WA, Berger C, Ruan M, Sprinkle M, Li X, Hu Y, et al. Large area and structured epitaxial graphene produced by confinement controlled sublimation of silicon carbide. Proceedings of the National Academy of Sciences. 2011;108(41):16900-16905. doi:http://dx.doi.org/10.1073/pnas.1105113108.

[6] Lin YM, Dimitrakopoulos C, Jenkins KA, Farmer DB, Chiu HY, Grill A, et al. 100-GHz Transistors from WaferScale Epitaxial Graphene. Science. 2010;327(5966):662-662. doi:http://dx.doi.org/10.1126/science.1184289. 
610

620

[13] Novoselov KS, Geim AK, Morozov SV, Jiang D, Zhang Y, Dubonos SV, et al. Electric Field Effect in Atomi-

[7] Tedesco JL, VanMil BL, Myers-Ward RL, McCrate JM, Kitt SA, Campbell PM, et al. Hall effect mobility of epitaxial graphene grown on silicon carbide. Applied Physics Letters. 2009;95(12):122102. doi:http://dx.doi.org/10.1063/1.3224887.

[8] Lin YM, Dimitrakopoulos C, Farmer DB, Han SJ, Wu Y, Zhu W, et al. Multicarrier transport in epitaxial multilayer graphene. Applied Physics Letters. 2010;97(11):112107. doi:http://dx.doi.org/10.1063/1.3485671.

[9] Tzalenchuk A, Lara-Avila S, Kalaboukhov A, Paolillo S, Syväjarvi M, Yakimova R, et al. Towards a quantum resistance standard based on epitaxial graphene. Nature nanotechnology. 2010;5(3):186-189. doi:http://dx.doi.org/10.1038/NNANO.2009.474.

[10] Riedl C, Coletti C, Iwasaki T, Zakharov AA, Starke U. Quasi-Free-Standing Epitaxial Graphene on $\mathrm{SiC}$ Obtained by Hydrogen Intercalation. Phys Rev Lett. 2009;103:246804. doi:http://dx.doi.org/10.1103/PhysRevLett.103.246804.

[11] de Heer WA, Berger C, Wu X, First PN, Conrad EH, Li X, et al. Epitaxial graphene. Solid State Communications. 2007;143(112):92 -100. doi:http://dx.doi.org/10.1016/j.ssc.2007.04.023.

[12] VanMil BL, Myers-Ward RL, Tedesco JL, Eddy CR Jr, Jernigan GG, Culbertson JC, et al. Graphene Formation on SiC Substrates. Materials Science Forum. 2009;615-617(1-2):211-214. 
cally Thin Carbon Films. Science. 2004;306(5696):666-669. doi:http://dx.doi.org/10.1126/science.1102896.

[18] Hass J, Varchon F, Millán-Otoya JE, Sprinkle M, Sharma N, de Heer WA, et al. Why Multilayer Graphene on 4H-SiC $(000 \overline{1})$ Behaves Like a Single Sheet of Graphene. Phys Rev Lett. 2008;100:125504. doi:http://dx.doi.org/10.1103/PhysRevLett.100.125504. 
655

[19] Hiebel F, Mallet P, Varchon F, Magaud L, Veuillen JY. Graphene-substrate interaction on $6 H-\mathrm{SiC}(000 \overline{1})$ : A scanning tunneling microscopy study. Phys Rev B. 2008;78:153412. doi:http://dx.doi.org/10.1103/PhysRevB.78.153412.

[20] Mathieu C, Barrett N, Rault J, Mi YY, Zhang B, de Heer WA, et al. Microscopic correlation between chemical and electronic states in epitaxial graphene on $\mathrm{SiC}(000 \overline{1})$. Phys Rev B. 2011;83:235436. doi:http://dx.doi.org/10.1103/PhysRevB.83.235436.

[21] Hass J, Feng R, Millán-Otoya JE, Li X, Sprinkle M, First PN, et al. Structural properties of the multilayer graphene/4H-SiC $(000 \overline{1})$ system as determined by surface x-ray diffraction. Phys Rev B. 2007;75:214109. doi:http://dx.doi.org/10.1103/PhysRevB.75.214109.

[22] Johansson LI, Watcharinyanon S, Zakharov AA, Iakimov T, Yakimova R, Virojanadara C. Stacking of adjacent graphene layers grown on C-face SiC. Phys Rev B. 2011;84:125405. doi:http://dx.doi.org/10.1103/PhysRevB.84.125405.

[23] Bouhafs C, Darakchieva V, Persson IL, Tiberj A, Persson PO, Paillet M, et al. Structural properties and dielectric function of graphene grown by high-temperature sublimation on $4 \mathrm{H}-\mathrm{SiC}(000-1)$. Journal of Applied Physics. 2015;117(8):085701. doi:http://dx.doi.org/10.1063/1.4908216.

[24] Nicotra G, Deretzis I, Scuderi M, Spinella C, Longo P, Yakimova $\mathrm{R}$, et al. Interface disorder probed at the atomic scale for 
graphene grown on the C face of SiC. Phys Rev B. 2015;91:155411. doi:http://dx.doi.org/10.1103/PhysRevB.91.155411.

[25] Johansson LI, Armiento R, Avila J, Xia C, Lorcy S, Abrikosov IA, et al. Multiple pi-bands and Bernal stacking of multilayer graphene on Cface $\mathrm{SiC}$, revealed by nano-Angle Resolved Photoemission. Sci Rep. 2014;4:4157. doi:http://dx.doi.org/10.1038/srep04157.

[26] Luxmi, Srivastava N, He G, Feenstra RM, Fisher PJ. Comparison of graphene formation on C-face and Si-face SiC $\{0001\}$ surfaces. Phys Rev B. 2010 Dec;82:235406. doi:http://dx.doi.org/10.1103/PhysRevB.82.235406.

[27] Hu Y, Ruan M, Guo Z, Dong R, Palmer J, Hankinson J, et al. Structured epitaxial graphene: growth and properties. Journal of Physics D: Applied Physics. 2012;45(15):154010. doi:http://dx.doi.org/10.1088/0022$3727 / 45 / 15 / 154010$.

[28] Yakimova R, Virojanadara C, Gogova D, Syväjarvi M, Siche D, Larsson K, et al. Analysis of the Formation Conditions for Large Area Epitaxial Graphene on $\mathrm{SiC}$ Substrates. Materials Science Forum. 2010;645-648(1-2):565-568. doi:http://dx.doi.org/10.4028/www.scientific.net/MSF.645-648.565.

[29] Ivanov IG, Hassan JU, Iakimov T, Zakharov AA, Yakimova R, Janzèn E. Layer-number determination in graphene on $\mathrm{SiC}$ by reflectance mapping. Carbon. 2014;77(0):492 - 500 . doi:http://dx.doi.org/10.1016/j.carbon.2014.05.054. 
[30] Sonde S, Giannazzo F, Raineri V, Yakimova R, Huntzinger JR, Tiberj A, et al. Electrical properties of the graphene $4 \mathrm{H}-\mathrm{SiC}(0001)$ interface probed by scanning current spectroscopy. Phys Rev B. 2009;80:241406. doi:http://dx.doi.org/10.1103/PhysRevB.80.241406.

[31] Kühne P, Herzinger CM, Schubert M, Woollam JA, Hofmann T. Invited Article: An integrated mid-infrared, far-infrared, and terahertz optical Hall effect instrument. Rev Sci Instrum. 2014;85:071301. doi:http://dx.doi.org/10.1063/1.4889920.

[32] Luxmi, Srivastava N, He G, Feenstra RM, Fisher PJ. Comparison of graphene formation on C-face and Si-face $\mathrm{SiC}\{0001\}$ surfaces. Phys Rev B. 2010;82:235406. doi:http://dx.doi.org/10.1103/PhysRevB.82.235406.

[33] Tedesco JL, Jernigan GG, Culbertson JC, Hite JK, Yang Y, Daniels KM, et al. Morphology characterization of argon-mediated epitaxial graphene on C-face SiC. Applied Physics Letters. 2010;96(22):222103. doi:http://dx.doi.org/10.1063/1.3442903.

[34] Bouhafs C, Stanishev V, Zakharov AA, Hofmann T, Khne P, Iakimov T, et al. Decoupling and ordering of multilayer graphene on C-face 3C-SiC(111). Applied Physics Letters. 2016;109(20). doi:http://dx.doi.org/10.1063/1.4967525.

[35] Srivastava N, He G, Luxmi, Mende PC, Feenstra RM, Sun Y. Graphene formed on $\mathrm{SiC}$ under various environments: comparison of $\mathrm{Si}$-face and C-face. Journal of Physics D: Applied Physics. 2012;45(15):154001. doi:http://dx.doi.org/10.1088/0022-3727/45/15/154001. 
[36] He G, Srivastava N, Feenstra RM. Formation of a Buffer Layer for Graphene on C-Face $\mathrm{SiC}\{0001\}$. J Electron Mat. 2013;43:819. doi:http://dx.doi.org/10.1007/s11664-013-2901-8.

[37] Yu YJ, Zhao Y, Ryu S, Brus LE, Kim KS, Kim P. Tuning the Graphene Work Function by Electric Field Effect. Nano Letters. 2009;9(10):34303434. doi:http://dx.doi.org/10.1021/nl901572a.

[38] Card HC, Rhoderick EH. Studies of tunnel MOS diodes I. Interface effects in silicon Schottky diodes. Journal of Physics D: Applied Physics. 1971;4(10):1589.

[39] Robertson J. High dielectric constant gate oxides for metal oxide $\mathrm{Si}$ transistors. Reports on Progress in Physics. 2006;69(2):327.

[40] Berciaud S, Ryu S, Brus LE, Heinz TF. Probing the Intrinsic Properties of Exfoliated Graphene: Raman Spectroscopy of Free-Standing Monolayers. Nano Letters. 2009;9(1):346-352. doi:http://dx.doi.org/10.1021/nl8031444.

[41] Kopylov S, Tzalenchuk A, Kubatkin S, Falko VI. Charge transfer between epitaxial graphene and silicon carbide. Applied Physics Letters. 2010;97(11):112109. doi:http://dx.doi.org/10.1063/1.3487782.

[42] Sidorov AN, Gaskill K, Buongiorno Nardelli M, Tedesco JL, Myers-Ward RL, Eddy CR, et al. Charge transfer equilibria in ambient-exposed epitaxial graphene on (0001) 6H-SiC. Journal of Applied Physics. 2012;111(11). doi:http://dx.doi.org/10.1063/1.4725413. 
745

755

[43] Mohiuddin TMG, Lombardo A, Nair RR, Bonetti A, Savini G, Jalil $\mathrm{R}$, et al. Uniaxial strain in graphene by Raman spectroscopy: G peak splitting, Grüneisen parameters, and sample orientation. Phys Rev B. 2009;79:205433. doi:http://dx.doi.org/10.1103/PhysRevB.79.205433.

[44] Mounet N, Marzari N. First-principles determination of the structural, vibrational and thermodynamic properties of diamond, graphite, and derivatives. Phys Rev B. 2005;71:205214. doi:http://dx.doi.org/10.1103/PhysRevB.71.205214.

[45] Das A, Pisana S, Chakraborty B, Piscanec S, Saha SK, Waghmare UV, et al. Monitoring dopants by Raman scattering in an electrochemically top-gated graphene transistor. Nature Nanotechnology. 2008;3(4):210215. doi:http://dx.doi.org/10.1038/nnano.2008.67.

[46] Lee JE, Ahn G, Shim J, Lee YS, Ryu S. Optical separation of mechanical strain from charge doping in graphene. Nat Commun. 2012;3:1024. doi:http://dx.doi.org/10.1038/ncomms2022.

[47] Biedermann LB, Bolen ML, Capano MA, Zemlyanov D, Reifenberger RG. Insights into few-layer epitaxial graphene growth on $4 H$-SiC $(000 \overline{1})$ substrates from STM studies. Phys Rev B. 2009 Mar;79:125411. doi:http://link.aps.org/doi/10.1103/PhysRevB.79.125411.

[48] Ferrari AC, Meyer JC, Scardaci V, Casiraghi C, Lazzeri M, Mauri F, et al. Raman Spectrum of Graphene and Graphene Layers. Phys Rev Lett. 2006;97:187401. doi:http://dx.doi.org/10.1103/PhysRevLett.97.187401. 
[49] Faugeras, $\mathrm{C}$ and Nerrire, $\mathrm{A}$ and Potemski, $\mathrm{M}$ and Mahmood, A and Dujardin, E and Berger, $\mathrm{C}$ and de Heer, W A . Few-

770

775

780 layer graphene on $\mathrm{SiC}$, pyrolitic graphite, and graphene: A Raman scattering study. Applied Physics Letters. 2008;92(1):011914. doi:http://dx.doi.org/10.1063/1.2828975.

[50] Li Y. Intrinsic Doping Dependence of Raman 2D Mode in Graphene: Signatures of Electron-Electron Interaction. In: Probing the Response of Two-Dimensional Crystals by Optical Spectroscopy. Springer Theses. Springer International Publishing; 2016. p. 9- 18. doi:http://dx.doi.org/10.1007/978-3-319-25376-3.

[51] Venezuela P, Lazzeri M, Mauri F. Theory of double-resonant Raman spectra in graphene: Intensity and line shape of defectinduced and two-phonon bands. Phys Rev B. 2011;84:035433. doi:http://dx.doi.org/10.1103/PhysRevB.84.035433.

[52] Sadowski ML, Martinez G, Potemski M, Berger C, de Heer WA. Landau Level Spectroscopy of Ultrathin Graphite Layers. Phys Rev Lett. 2006;97:266405. doi:http://dx.doi.org/10.1103/PhysRevLett.97.266405.

[53] Orlita M, Faugeras C, Grill R, Wysmolek A, Strupinski W, Berger C, et al. Carrier Scattering from Dynamical Magnetoconductivity in Quasineutral Epitaxial Graphene. Phys Rev Lett. 2011;107:216603. doi:http://dx.doi.org/10.1103/PhysRevLett.107.216603.

[54] Kühne P, Darakchieva V, Yakimova R, Tedesco JD, Myers-Ward RL, Eddy CR, et al. Polarization Selection Rules for Inter- 
Landau-Level Transitions in Epitaxial Graphene Revealed by the Infrared Optical Hall Effect. Phys Rev Lett. 2013;111:077402. doi:http://dx.doi.org/10.1103/PhysRevLett.111.077402.

[55] Maëro S, Torche A, Phuphachong T, Pallecchi E, Ouerghi A, Ferreira R, et al. Disorder-perturbed Landau levels in highelectron-mobility epitaxial graphene. Phys Rev B. 2014;90:195433. doi:http://dx.doi.org/10.1103/PhysRevB.90.195433.

[56] Camara N, Rius G, Huntzinger JR, Tiberj A, Magaud L, Mestres N, et al. Early stage formation of graphene on the C face of 6H-SiC. Applied Physics Letters. 2008;93(26):263102. doi:http://dx.doi.org/10.1063/1.3056655. 